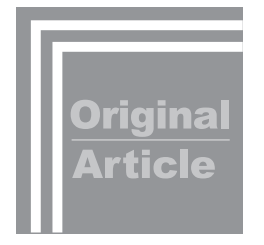

\title{
Usefulness of a Microcatheter with a Short and Acute Angled Tip Made from an Excelsior SL-10 Pre-shaped S Catheter Preserving Its Distal Curve
}

Katsumi Hoya, ${ }^{1}$ Kazuya Nakamura, ${ }^{2}$ Hajime Nishido, ${ }^{1}$ Akihiro Nishikawa, ${ }^{2}$ Kenta Ohara, ${ }^{1}$ Shinya Miyamoto, ${ }^{1}$ and Kenji Wakui ${ }^{2}$

Objective: A method to create a microcatheter with a short and acute-angled tip using an Excelsior SL-10 pre-shaped $S$ catheter (Stryker, Kalamazoo, MI, USA) by straightening its proximal curve is described. Its clinical application, in which a guidewire and a microcatheter are led into a distal artery branching sharply to prepare stent deployment, is presented. Methods: The proximal curve of a catheter was introduced into a disposable 18-gauge blunt needle. The needle was heated using a heat gun so as to heat and straighten only the proximal curve and to preserve the original shape and firmness of the distal curve. The catheter was then used to lead a guidewire to a sharply branching distal artery and subsequently to navigate itself into the artery. In each case, the angle formed by the vector of the proximal parent artery and that of the distal artery was measured to evaluate the technical difficulty. The usefulness of the catheter was assessed by whether stenting was completed.

Results: The microcatheter was used in 10 cases. The average angle between the distal artery and the proximal parent artery was $144^{\circ}$. In two cases where the angle was over $180^{\circ}$, the guidewire could not enter the orifice of the distal artery directly. The guidewire could directly enter the orifice of the distal branch in eight cases, with angles of $108^{\circ}-151^{\circ}$. In seven of the eight, the guidewire and microcatheter could go further into the distal artery, and stenting was achieved. Conclusion: A short and acutely angled catheter tip made from an Excelsior SL-10 pre-shaped S catheter with preservation of the distal curve is stable in shape. It is often useful when leading a guidewire to an artery branching at a sharp angle to prepare for stent-assisted coiling.

Keywords > aneurysm, microcatheter, shaping, stent

\section{Introduction}

The shape of a microcatheter tip is important for neuroendovascular therapy. A short and acutely angled tip is difficult to make from braided microcatheters by steam shaping, and we often find that it easily becomes blunt by guidewire

${ }^{1}$ Department of Neurosurgery, Teikyo University Chiba Medical Center, Ichihara, Chiba, Chiba, Japan

${ }^{2}$ Chiba Neurosurgical Clinic, Chiba, Chiba, Japan

Received: April 15, 2019; Accepted: August 20, 2019

Corresponding author: Katsumi Hoya. Department of Neurosurgery, Teikyo University Chiba Medical Center, 3426-3 Anesaki, Ichihara, Chiba, Chiba 299-0111, Japan

Email:khoya@med.teikyo-u.ac.jp

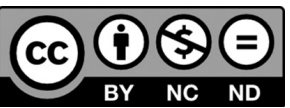

This work is licensed under a Creative Commons Attribution-NonCommercialNoDerivatives International License.

(C)2019 The Japanese Society for Neuroendovascular Therapy insertion through the catheter. ${ }^{1} \mathrm{~A}$ heat gun gives higher heat than steam and enables us to make a small catheter tip stiffer. ${ }^{2)}$ However, over-heating breaks microcatheters. ${ }^{3)}$ In this report, a new method to make a short and acutely angled tip by straightening a proximal curve of an Excelsior SL-10 pre-shaped S catheter (Stryker, Kalamazoo, MI, USA) is introduced. The original firmness is kept in the distal curve of the pre-shaped catheter. This catheter tip is used to lead a guidewire and the microcatheter itself into a distal artery branching at an acute angle from an aneurysmal neck without entering the aneurysmal dome. This report shows that the catheter is useful to navigate it safely into a distal artery when stent deployment is planned for coil embolization.

\section{Materials and Methods}

The inner lumen of the pre-shaped S catheter is flushed by heparinized saline. A guidewire is inserted reversely from 


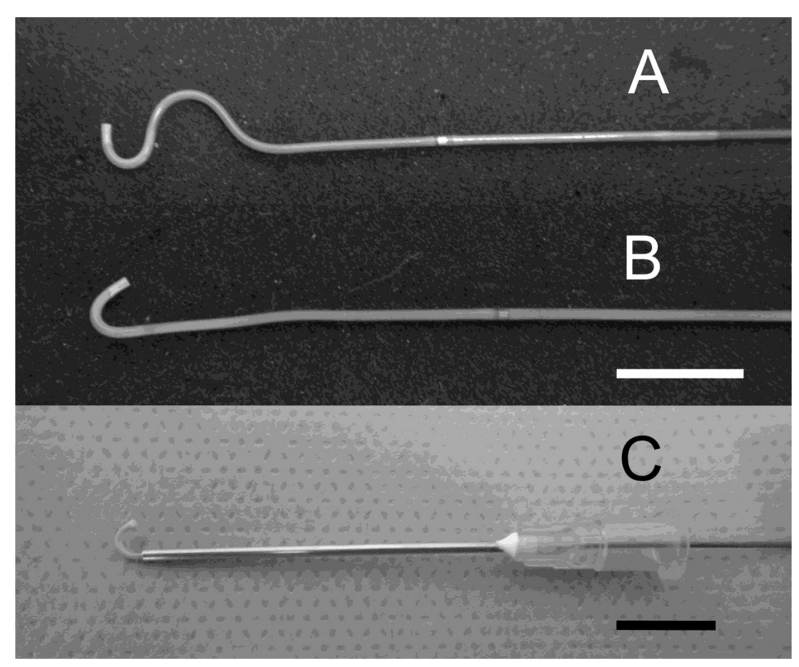

Fig. 1 (A) Original shape of the Excelsior SL-10 pre-shaped S catheter. (B) The catheter whose proximal curve is straightened with preservation of the distal curve. (C) A disposable 18-gauge blunt needle and the distal curve of the microcatheter pushed out of the blunt needle. The white bar (A and $\mathbf{B})$ and the black bar $(\mathbf{C})=1 \mathrm{~cm}$.

the proximal end of a catheter. The tip of the catheter is temporarily straightened by the wire and introduced into a disposable 18-gauge blunt needle (JMS, Hiroshima, Japan), which is flushed by saline beforehand. A silver irrigation needle (Fujita Medical Instruments, Tokyo, Japan) or a neurosurgical suction cannula with a small caliber can be a substitute for an 18-gauge needle. The distal curve of the catheter is pushed out of the blunt needle. The proximal end of the guidewire stays in the proximal curve of the $\mathrm{S}$ as a straight mandrel. An 18-gauge needle is heated with a heat gun (Robert Bosch, Gerlingen, Germany) set at $140^{\circ} \mathrm{C}$ at the position approximately $2 \mathrm{~cm}$ from the hot air outlet, where the actual temperature is $100^{\circ} \mathrm{C}-110^{\circ} \mathrm{C}$, for $60-90$ seconds. The tip of the microcatheter outside of the needle is not heated directly to keep its pre-shaped form. The newly shaped catheter is shown in Fig. 1. The proximal curve of the catheter is directly heated for 30 seconds, protecting the distal curve if straightening is insufficient or if it is necessary to re-shape it to fit vascular anatomy. The inner lumina of needles or cannulae can be easily crushed by bending. They can be used only for straightening, not for re-shaping.

Patients in whom this type of microcatheter was used from July 2017 to November 2018 were studied. This catheter was applied for stent deployment in which a microcatheter first needs to pass across an aneurysmal orifice.

This study retrospectively evaluated the usefulness of this newly shaped catheter. Angiograms including 3D

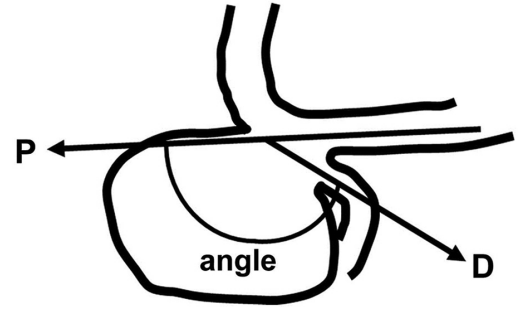

Fig. 2 An illustration showing how to measure the angle between the vector of the proximal parent artery $(\mathbf{P})$ and that of the distal artery (D) where stenting started.

images were reviewed for aneurysmal locations and the arterial anatomy around them. The angle formed by the vector of the proximal parent artery and that of the distal branch was measured on a 3D image to evaluate technical difficulty (Fig. 2). Operation records were examined for technical achievement, that is, the guidewire and microcatheter could be led directly into the sharply branching distal artery, and stenting was completed. The time taken to manipulate a guidewire into a distal artery and subsequently navigate a microcatheter was also measured.

\section{Results}

This type of microcatheter was used in 10 cases where the distal artery branched at an acute angle, and stent-assisted coil embolization was intended. Aneurysmal locations were the internal carotid artery (ICA)- posterior communicating artery bifurcation (IC-P-com) in three cases, the anterior communicating (A-com) in two, the middle cerebral artery (MCA) bifurcation in three cases, and the basilar bifurcation in two cases (Table 1). In these cases, the aneurysmal neck was broad, and the distal artery branched at an acute angle from a proximal parent artery. The average angle between the vector of the proximal parent artery and that of the distal artery where stenting started was $144^{\circ}\left(108^{\circ}-201^{\circ}\right)$. In two cases, the angle was over $180^{\circ}$, which means the distal branch turned in the completely opposite direction. Angiograms of illustrative cases are shown in Figs. 3 and $\mathbf{4}$.

The plan was to direct the catheter tip to the acutely angled distal artery and to make the guidewire turn the acute angle and enter the distal artery directly, without entering the aneurysmal dome. When a 0.012 or 0.014 -inch wire was used, the wire opened the curve of the catheter tip wider and could not enter into the distal artery. The procedure was successful when wires with softer tips, a 0.010-14 TENROU $200 \mathrm{~cm}$ (Kaneka Medix, Osaka, Japan) 
Table 1 Data of angiography and endovascular treatment

\begin{tabular}{|c|c|c|c|c|c|c|}
\hline $\begin{array}{l}\text { Case } \\
\text { No. }\end{array}$ & $\begin{array}{l}\text { Aneurysmal } \\
\text { location }\end{array}$ & $\begin{array}{l}\text { Angle between the } \\
\text { proximal parent } \\
\text { artery and the distal } \\
\text { branch }\left(^{\circ}\right)\end{array}$ & Re-shaping & $\begin{array}{l}\text { Technical } \\
\text { success }\end{array}$ & $\begin{array}{l}\text { Time for leading a } \\
\text { GW and a MC into } \\
\text { a distal artery } \\
\text { (seconds) }\end{array}$ & \\
\hline 1 & IC-Pcom & 140 & No & Yes & 240 & \\
\hline 2 & MCA bifurcation & 151 & Yes & Yes & 126 & \\
\hline 3 & A-com & 108 & No & Yes & 300 & \\
\hline 4 & BA top & 123 & No & Yes & 122 & \\
\hline 5 & A-com & 140 & No & Yes & 75 & \\
\hline 6 & BA top & 128 & No & Yes & 70 & \\
\hline 7 & MCA bifurcation & 135 & No & Yes & 126 & \\
\hline 8 & MCA bifurcation & 201 & Yes & No & n.a. & $\begin{array}{l}\text { Leading a GW into } \\
\text { the distal branch was } \\
\text { impossible }\end{array}$ \\
\hline 9 & IC-Pcom & 185 & Yes & No & n.a. & $\begin{array}{c}\text { Leading a GW into } \\
\text { the distal branch was } \\
\text { impossible }\end{array}$ \\
\hline 10 & IC-Pcom & 117 & Yes & No & n.a. & $\begin{array}{l}\text { A GW entered the } \\
\text { distal branch orifice } \\
\text { and did not go distally } \\
\text { enough to lead a MC } \\
\text { into it for stenting }\end{array}$ \\
\hline
\end{tabular}

A-com: anterior communicating artery; BA: basilar artery; GW: guidewire; IC-Pcom: internal carotid artery-posterior communicating artery; MC: microcatheter; MCA: middle cerebral artery; n.a.: not assessed

or a 0.010 CHIKAI $200 \mathrm{~cm}$ (Asahi Intecc Co., Ltd, Aichi, Japan), were used. After the wire tip was sent distally, a microcatheter was led along the wire and passed across the aneurysmal orifice to prepare stent deployment over it. The time taken to lead a guidewire and a catheter into a distal artery ranged from 70 to 300 seconds in the successful cases. The average was 151 seconds. The time did not depend on the difficulty in leading a guidewire into the orifice of the distal artery. It mainly depended on the difficulty in navigating a wire further in the branch.

Neuroform Atlas (Stryker) stents were used because they were the only stent products which can be inserted into SL-10 in Japan and suitable for deployment at an acute angle. This stenting plan was completed in seven cases.

In one of the three unsuccessful cases (Case 10), the catheter tip was successfully placed into the orifice of the distal artery. The guidewire could not go through a tight curve just distal to the orifice, and the microcatheter could not go further. Stenting was abandoned, and the coiling was performed using microcatheter protective technique. ${ }^{4}$ )

In the other two of three cases (Cases 8 and 9) where the branching angle was over $180^{\circ}$, many kinds of guidewires were tried, and none of them could turn the acute angle and enter the distal artery. A loop was formed within the aneurysms with the wire and microcatheter, and the distal artery entered. Thereafter, the catheter was pulled and straightened at the neck, and stenting was completed.

In four of the 10 cases, the catheter tip tended to be positioned in an unintended direction. A straightened part of the catheter was re-shaped to fit the curve of the proximal artery, mostly bent in the same direction as the distal curve of the catheter.

\section{Discussion}

Today, we usually use braided microcatheters. The product instructions of the microcatheters state that their tips should be shaped by steaming. However, we often find that those shaped in such a manner are easy to blunt ${ }^{1,5}$ and it is very difficult to make them when they need to be short and acutely angled. Heat guns, work tools supplying hot air, give higher heat than steam and are recently being used for this purpose. Matsumoto et al. reported a method to create a very small tip, which they used mainly for coiling a small aneurysm. ${ }^{2)}$ They successfully shaped the tip of a straight type SL-10 microcatheter or Headway microcatheter (MicroVention TERUMO, Tustin, CA, USA) using a 


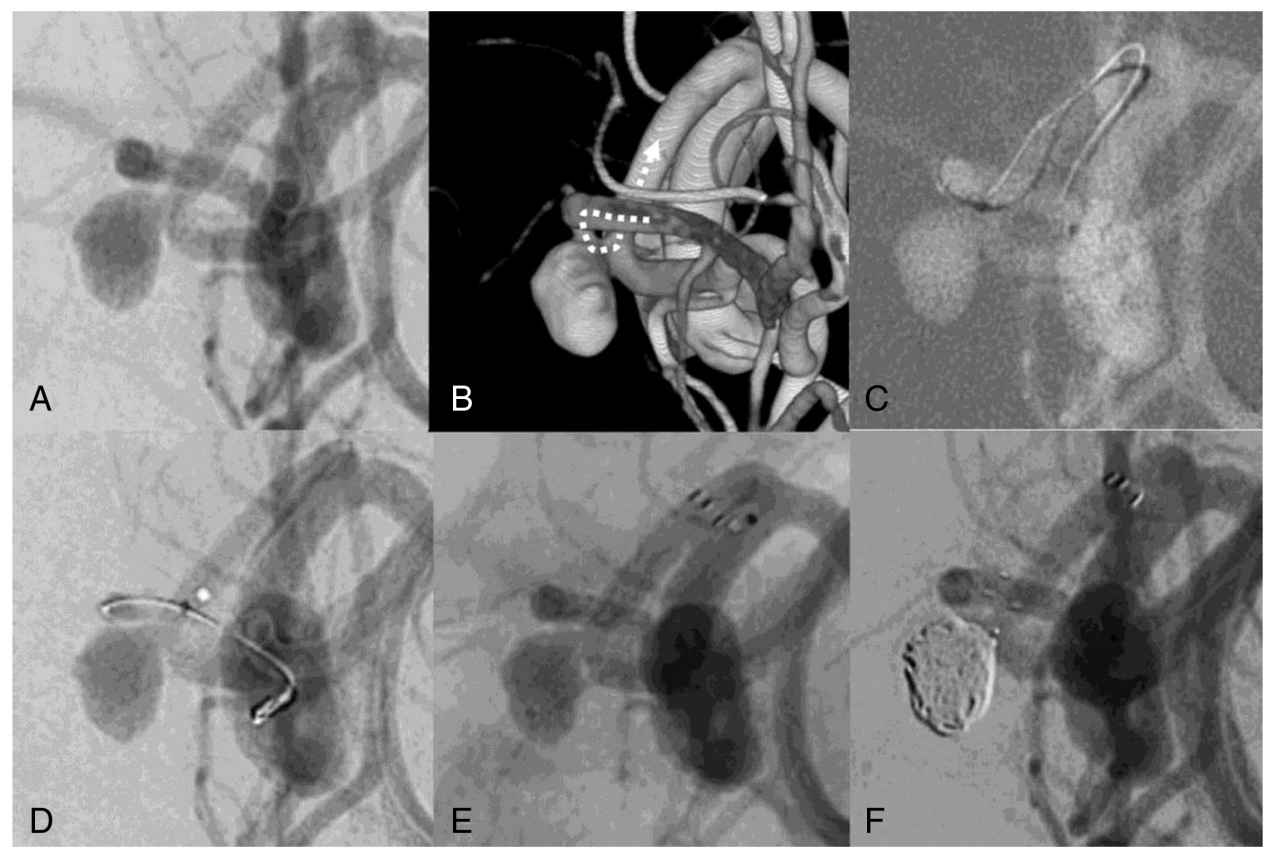

Fig. 3 Case 5 of an unruptured A-com artery aneurysm. (A) Pre-operative left carotid angiogram. (B) 3D image of the aneurysm. A broken line arrow shows the plan to deploy a Neuroform Atlas (Stryker, Kalamazoo, MI, USA) stent from right A2 to left A1 to protect A-com from coil loops. (C) A 0.010-14 TENROU wire (Kaneka Medix, Osaka, Japan) successfully enters right A2. (D) The wire is navigated distally, and the microcatheter can follow it into right A2. (E) A Neuroform Atlas stent is deployed. (F) Postoperative angiogram. A-com: anterior communicating

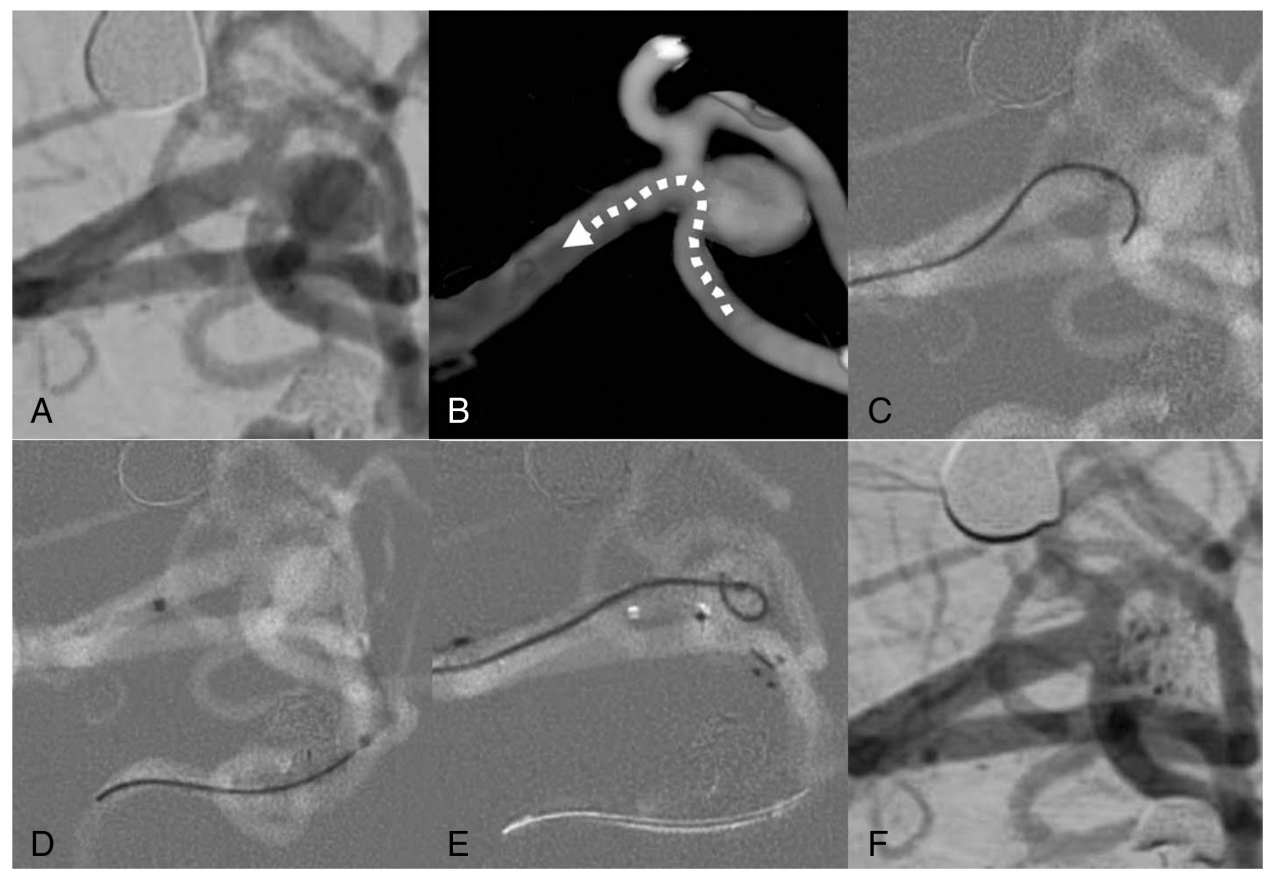

Fig. 4 Case 7, an unruptured left middle cerebral artery bifurcation aneurysm. (A) Preoperative left carotid angiogram. (B) 3D image of the aneurysm. A broken line arrow shows the plan to deploy a Neuroform Atlas (Stryker, Kalamazoo, MI, USA) stent from M2 (the inferior trunk) to M1. (C) A 0.010-14 TENROU wire (Kaneka Medix, Osaka, Japan) enters the inferior trunk. (D) The wire is led distally, and the microcatheter can follow it (E) The microcatheter is pulled to deploy a Neuroform Atlas stent. (F) Postoperative angiogram. 
mandrel with a loop of very short diameter and heating it with a heat gun. Adachi et al. reported that the material of a Headway microcatheter is denatured by hot air with a temperature over $110^{\circ} \mathrm{C} .{ }^{3)}$ Today, there are no exact official data concerning the optimal temperature and duration of heating for microcatheters when using a heat gun. Higher heating may make a microcatheter stiffer, but over-heating breaks microcatheters.

Kiyosue et al. showed that the consistency of the tip angle of pre-shaped microcatheters (Prowler Plus, Cordis Endovascular Systems, Miami, FL, USA) was higher than that of steam-shaped angles of the same brand. ${ }^{5}$ Preshaped SL-10 catheters are also products that are firmly shaped by a designated heating process, and their shapes are stable during endovascular procedures. In several types of pre-shaped SL-10s, the pre-shaped S catheter has a short and acute-angled tip in the distal portion. If the proximal curve of the catheter fits the arterial anatomy, the original catheter is very useful for coiling small aneurysms or directing a guidewire to a distal artery branching at an acute angle. However, when it does not fit the arterial anatomy, the short tip is misdirected and does not work well.

In order to use only the short and acutely angled tip of the catheter, the proximal curve of a pre-shaped $\mathrm{S}$ was straightened by inserting it into a disposable 18-gauge blunt needle and by a straight mandrel of a proximal guidewire end. The heating conditions including additional heating were milder than those reported by Matsumoto et al. ${ }^{2)}$ In our experience, microcatheters were not denatured and could be used safely. When the temperature of the heated needle was measured, it actually did not exceed $100^{\circ} \mathrm{C}$. When steaming a blunt needle instead of using a heat gun was tested, it achieved the same effect. The straightened part was stable in shape after heating. We do not think that the method presented in this paper is the only one to make such a microcatheter. The important point is to avoid heating the distal curve of the $\mathrm{S}$ and to preserve its firmness.

It is sometimes difficult to navigate a guidewire and a microcatheter to a distal artery branching at an acute angle from an aneurysmal neck. One of the known methods is to form a loop within the aneurysm with a guidewire and a microcatheter and enter the distal artery. ${ }^{6-8)}$ This method should be avoided if possible because it may apply excessive stress to the aneurysmal wall and cause a fatal rupture. The catheter presented was often useful and safe to pass across an aneurysmal orifice and to deploy a Neuroform Atlas stent.
The procedure was unsuccessful in three cases. In two of the three (Cases 8,9), the angles between the parent artery and the distal branch were over $180^{\circ}$. Though pre-shaped SL-10 catheters are products that are firmly shaped, shape stability was limited and insufficient to make the wire tip turn to the opposite direction. A more acutely angled catheter tip may be created with the method reported by Matsumoto et al. ${ }^{2}$ However, the durability of its shape when the catheter was used for leading a guidewire into a distal artery to prepare stent deployment has not been reported and is unknown today.

In the other case (Case 10), the catheter tip successfully led a guidewire to the orifice of the distal artery but was not supportive enough for further guidewire manipulation. In this case, changing the shape of the wire tip or use of more flexible guidewires might have led to better results. This is a subject for future analysis.

\section{Conclusion}

A new method to make a short and acutely angled catheter tip from an SL-10 pre-shaped S catheter by straightening its proximal curve and preserving its distal curve firmness was presented. The catheter was often useful to lead a guidewire and the microcatheter itself safely to a distal artery branching at an acute angle from an aneurysmal neck when stent-assisted coiling was planned.

\section{Disclosure Statement}

All authors have no conflict of interest.

\section{References}

1) Abe $T$, Hirohata $M$, Tanaka $N$, et al: Distal-tip shapeconsistency testing of steam-shaped microcatheters suitable for cerebral aneurysm coil placement. AJNR Am J Neuroradiol 2004; 25: 1058-1061.

2) Matsumoto $H$, Nishiyama $H$, Tetsuo $Y$, et al: Ultra-small catheter shaping method with a sheath dilator: usefulness for coil embolization of cerebral aneurysms. JNET 2019; 13: 21-27.

3) Adachi A, Kobayashi E, Kado K, et al: The optimal conditions for microcatheter shaping. JNET 2016; 10: 236-242.

4) Lee JY, Seo JH, Cho YD, et al: Endovascular treatment of wide-neck intracranial aneurysms using a microcatheter protective technique: results and outcomes in 75 aneurysms. AJNR Am J Neuroradiol 2011; 32: 917-922. 
5) Kiyosue H, Hori $Y$, Matsumoto S, et al: Shapability, memory, and luminal changes in microcatheters after steam shaping: a comparison of 11 different microcatheters. AJNR Am J Neuroradiol 2005; 26: 2610-2616.

6) Snyder KV, Natarajan SK, Hauck EF, et al: The balloon anchor technique: a novel technique for distal access through a giant aneurysm. J Neurointerv Surg 2010; 2: 363-367.
7) Effendi K, Sacho RH, Belzile F, et al: The wire anchor loop traction (WALT) maneuver. J Neurointerv Surg 2016; 8:e7.

8) Kanenaka N, Sato H, Abe H, et al: [Exchange technique using flow directed microcatheter during difficulty in navigation of delivery catheter in stent-assisted coil embolization]. JNET 2014; 8: 107-113 (in Japanese). 